\section{Amyloid myopathy: a diagnostic challenge}

\author{
Heli Tuomaala, ${ }^{1,2}$ Mikko Kärppä, \\ Hannu Tuominen, ${ }^{3}$ Anne M. Remes, ${ }^{1,2}$
}

\author{
'Department of Neurology, University of \\ Oulu, ${ }^{2}$ Clinical Research Center, Oulu \\ University Hospital, ${ }^{3}$ Department of \\ Pathology, University of Oulu, Oulu, \\ Finland
}

\begin{abstract}
Amyloid myopathy (AM) is a rare manifestation of primary systemic amyloidosis (AL). Like inflammatory myopathies, it presents with proximal muscle weakness and an increased creatine kinase level. We describe a case of AL with severe, rapidly progressive myopathy as the initial symptom. The clinical manifestation and muscle biopsy were suggestive of inclusion body myositis. AM was not suspected until amyloidosis was seen in the gastric mucosal biopsy. The muscle biopsy was then re-examined more specifically, and Congo red staining eventually showed vascular and interstitial amyloid accumulation, which led to a diagnosis of AM. The present case illustrates the fact that the clinical picture of AM can mimic that of inclusion body myositis.
\end{abstract}

\section{Introduction}

Primary systemic amyloidosis (AL) is a rare disease. Its typical presentations are nephrotic syndrome, cardiomyopathy, peripheral neuropathy and hepatomegaly. ${ }^{1,2}$ One uncommon manifestation is amyloid myopathy (AM), the symptoms of which are usually non-specific, typically including progressive proximal weakness with an increased creatine kinase (CK) level, macroglossia and muscle pseudohypertrophy. ${ }^{3,45}$ Muscle weakness is atypical as the initial symptom of the disease. ${ }^{1,6}$ The diagnosis of AM is usually overlooked and it is often misdiagnosed as inflammatory myopathy, even when a muscle biopsy is available. ${ }^{7}$ When suspecting AM, Congo red staining and an immunohistochemistry or immunofluoresence assay should be performed. Furthermore, the use of MRI for evaluating neuromuscular disorders and inflammatory myopathies has become increasingly common, especially in cases of polymyositis. ${ }^{89}$ We present here a case of AL with severe and rapidly progressive myopathy as the initial symptom.

\section{Clinical summary}

The patient was a 65 -year-old man with a history of hypertension and obesity (weight $144 \mathrm{~kg}$ ) who presented with proximal muscle weakness in the legs and a $35 \mathrm{~kg}$ weight loss. No familial occurrence of muscle diseases was reported. At the first clinical examination after two years of experiencing fatigue, the patient showed slight proximal muscle weakness corresponding to $4 / 5$ on the Medical Research Council (MRC) scale and minor weakening of the tendon reflexes but was able to walk without support. Muscle weakness progressed within six months, however, forcing the patient to use a walking aid. He then had grade 3 symptoms on the MRC scale in the shoulder girdle and bilaterally in the thigh muscles, with grade 4 in the distal hand and leg muscle groups. In addition, the new findings included mild dysphagia, macroglossia and an absence of tendon reflexes. No muscle atrophy was established and sensory functions were intact. The results of brain MRI and a cerebrospinal fluid analysis were normal.

Laboratory findings included elevated serum creatine kinase (CK) and serum aldolase levels (Table 1). Liver function tests were abnormal, but MCV was slightly elevated. Serum transferrine, protein electrophoresis and immunofixation findings were normal, whilst serum ferritine was elevated. The plasma albumin level was low, and marked proteinuria was observed. An electromyography (EMG) revealed proximal fibrillation and motor unit potentials of short duration, low amplitude and a polyphasic character and insertional activity. The myopathy specific EMG findings aroused suspicions of inclusion body myositis and polymyositis. The patient had arrhythmic episodes of supraventricular tachycardia (SVT), and a chest X-ray film showed cardiac dilatation. Echocardiography revealed marked left ventricular hypertrophy. The ejection fraction was $55 \%$, and the myocardium was slightly abnormal, indicating muscle disease.

Muscle magnetic resonance imaging (MRI) was performed with T1, short tau inversion recovery (STIR) and T2 FAT SAT axial projections (Figure 1). Axial T2 fat suppression magnetic resonance imaging of the thighs showed a mildly increased signal intensity in the posterior muscles, while coronal T2 fat suppression magnetic imaging showed a coarse reticular pattern in the subcutaneous fat of the lower extremities. A left vastus lateralis muscle biopsy showed mainly non-specific changes, but there was some evidence suggestive of inclusion body myositis. An abdominal ultrasonography showed the presence of a slightly clear liver. The abdominal CT results were normal. The provisional diagnosis at this stage was considered to be inclusion body
Correspondence: Anne M. Remes

Department of Neurology, University of Oulu Box 5000, FIN-90014 0ulu, Finland

E-mail: anne.remes@oulu.fi

Key words: amyloid myopathy, amyloidosis, congo red, magnetic resonance imaging, myopathy

Acknowledgments: this work was supported financially in part by grants from the Finnish Medical Foundation (AMR) and the Päivikki and Sakari Sohlberg Foundation (AMR).

Contributions: HT participated in designing the study and drafted the manuscript, MK participated in examining the patient, designing the study and revising the manuscript, and HT carried out the neuropathological examinations and helped to revise the manuscript. AMR participated in designing and coordinating the study and helped to draft the manuscript. All the authors have read and approved the final manuscript.

Conflict of interest: the authors reported no potential conflicts of interests.

Received for publication: 26 February 2009.

Revision received: 8 June 2009.

Accepted for publication: 11 June 2009.

This work is licensed under a Creative Commons Attribution 3.0 License (by-nc 3.0)

(C) Copyright H. Tuomaala et al., 2009

Licensee PAGEPress, Italy

Neurology International 2009; 1:e7

doi:10.4081/ni.2009.e7

myositis. A gastroscopy was performed on account of elevated liver enzymes and a suspicion of celiac disease, and biopsies from the duodenum, antrum and corpus revealed diffuse mucosal, submucosal and vascular amyloid accumulation. The histological findings conformed to primary amyloidosis. Immunofixation of serum and urine also revealed a minimal amount of lambda light chains. The amount of plasma cells in a bone marrow biopsy was normal. In view of the gastric biopsy findings and the diagnosis of primary amyloidosis, the muscle biopsy was re-examined more specifically. Congo red staining eventually showed vascular and extracellular amyloid accumulation, which led to a diagnosis of AM. Despite the gastrointestinal changes, the patient did not have any clinical symptoms of this.

After the IBM diagnosis, the patient was treated with prednisone $100 \mathrm{mg} /$ day for two months and then at a decreasing dosage for three months. Since the muscle weakness had markedly progressed in spite of this prednisone medication, the treatment was stopped after five months. Immunoglobulin $0.4 \mathrm{mg} / \mathrm{kg}$ i.v. was given for five days, leading to a minor improvement in muscle strength, so that the 
treatment was repeated three times monthly. oral melphalan and prednisone treatment was also started after the diagnosis of amyloid myopathy, the total target dose of melphalan being $740 \mathrm{mg}$, while prednisone was given 100 mg p.o. once a day for four days. Only a minor response to the medication was seen as far as the progression of muscle weakness and other symptoms during one year of follow-up was concerned. The patient died of pneumonia at the age of 69 years. No autopsy was performed.

\section{Pathological findings}

The first examination of the left vastus lateralis muscle biopsy showed changes suggesting neurogenic atrophy, regeneration and some rimmed vacuoles. NADH-Tr and trichrome staining results were normal, but SDH-COX staining revealed some COX-negative fibers. No MCH-class I upregulation was seen on the muscle membranes nor any fiber necrosis. Although the muscle biopsy findings were mainly non-specific, there was some evidence suggestive of inclusion body myositis.

A gastroscopy was performed later on account of elevated liver enzymes and a suspicion of celiac disease, and duodenum, antrum and corpus biopsies revealed diffuse mucosal, submucosal and vascular amyloid accumula-
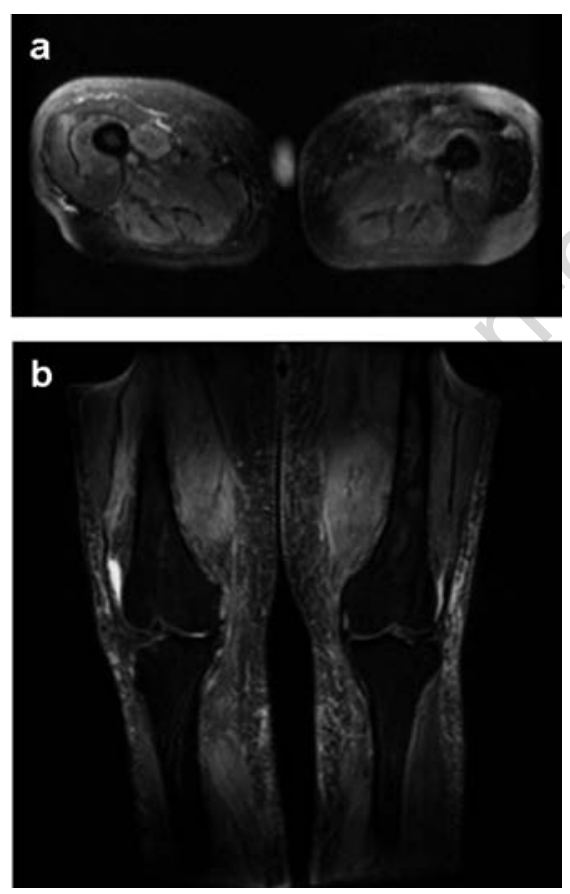

Figure 1. (a) Axial T2 fat suppression magnetic resonance imaging of the patient's thighs. Mildly increased signal intensity is visible in the posterior muscles. (b) Coronal T2 fat suppression magnetic imaging show a coarse reticular pattern in the subcutaneous fat of the lower extremities.
Table 1. Abnormal laboratory test findings.

\begin{tabular}{lcc}
\hline Laboratory test & Laboratory values & Reference values \\
S-CK (U/L) & $1900-2200$ & $40-280$ (for men) \\
S-aldolase (U/L) & 18 & $<5$ \\
\hline E-MCV (fL) & $100-106$ & $82-98$ \\
P-albumin (g/L) & $24-35$ & $36-45$ \\
\hline U-protein (mg/L) & 2100 & $<200$ \\
S- ferritine (ug/L) & 797 & $20-250$ \\
\hline P-ALAT (U/L) & $90-196$ & $10-70$ \\
P-GT (U/L) & $242-1284$ & $15-115$
\end{tabular}

tion which was resistant to potassium permanganate digestion. An immunohistochemical assay was negative for amyloid A, but the accumulation was positive for lambda light chain staining. The histological findings conformed to primary amyloidosis. The number of plasma cells in a bone marrow biopsy was normal. In view of the gastric biopsy findings and the diagnosis of primary amyloidosis, the muscle biopsy was examined again more specifically. Congo red staining eventually showed vascular and extracellular amyloid accumulation, which led to a diagnosis of AM (Figure 2). An immunohistochemical assay for amyloid A was negative, and kappa and lambda light chain stainings were inconclusive.

\section{Discussion}

The first recognised patient presenting with amyloid involvement in muscle, the condition known as AM, was reported by Lubarsch in $1929,{ }^{10}$ and it has now been reported in three of the largest series that myopathy is rarely caused by amyloidosis. ${ }^{14,6,7}$ The symptoms of AM are usually non-specific, including progressive proximal weakness with an elevated creatine kinase level, macroglossia and muscle pseudohypertrophy as the typical features. ${ }^{3,45} \mathrm{We}$ described a case of $\mathrm{AL}$ with severe, rapidly progressive myopathy as the initial symptom. The patient had suffered from mild muscle weakness for 2.5 years, followed by a rapid progression of the condition and the onset
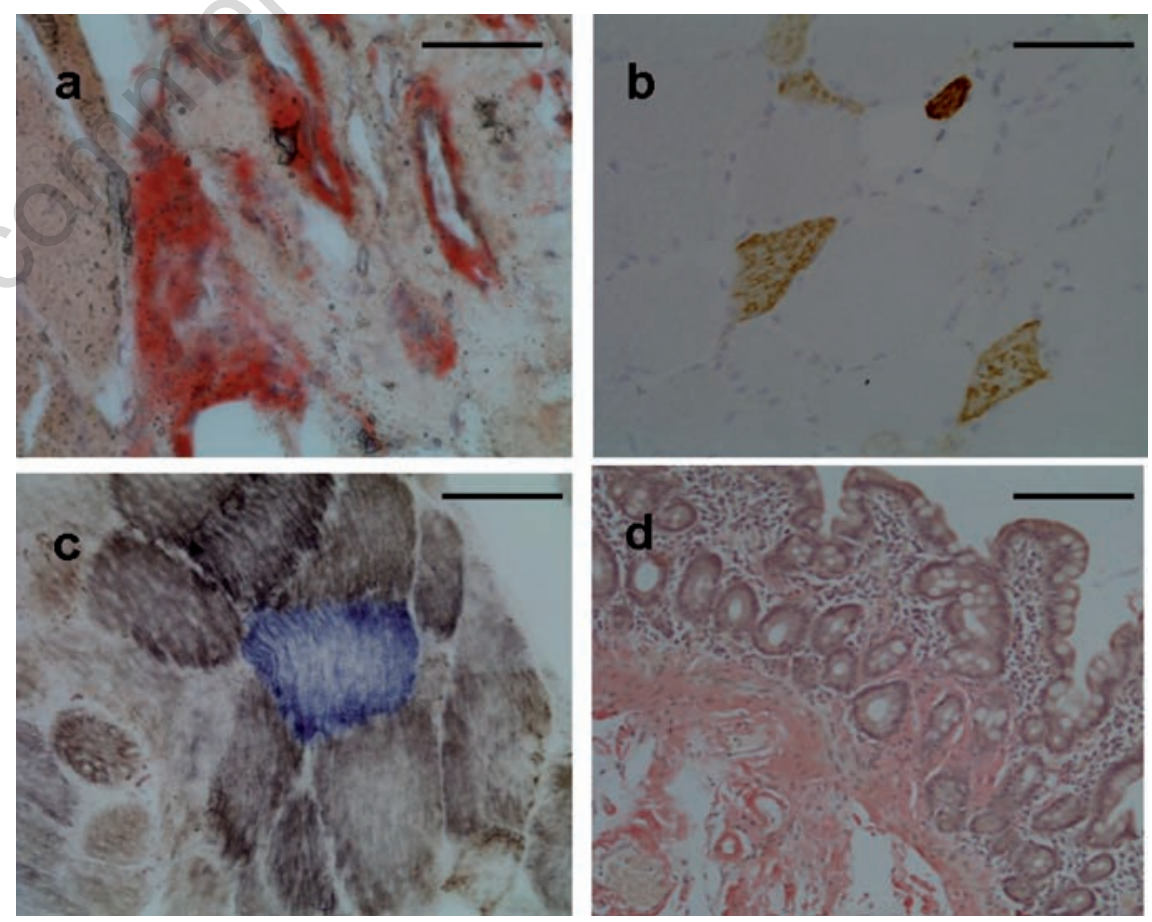

Figure 2. (a) Biopsy of the vastus lateralis muscle shows the vascular wall and an interstitial accumulation of amyloid (Congo red), (b) expression of neonatal myosin in atrophic cells (myosin heavy chain neonatal immunoperoxidase staining, original magnification $x$ 20), (c) a COX-negative fiber (succinate dehydrogenase - cytochrome oxidase double staining) and (d) a duodenal mucosal biopsy containing amyloid (Congo red). Scale bar $100 \mu \mathrm{m}$ (figure a-c), $200 \mu \mathrm{m}$ (figure d). 
of other clinical symptoms such as dysphagia and macroglossia. Visceral manifestations of AL, including cardiomyopathy, nephropathy and gichanges, were also observed. Weakness of knee extensors with absent knee reflexes was prominent in the early course of the disease suggesting the diagnosis of IBM although distal involvement in the hands was observed later.

Although amyloid myopathy is a welldescribed disorder, it is often overlooked, for two reasons: first, it is rarely observed clinically, and second, a broad span of differential diagnoses is needed. It is usually misdiagnosed as inflammatory myopathy, even given a muscle biopsy, as seen here and reported by others. Three cases of AM mimicking polymyositis have been reported lately, all showing similarities in clinical, neurophysiological and muscle biopsy findings when stained only with hematoxylin-eosin. In all three cases, the original muscle biopsy samples were analyzed retrospectively with Congo red staining, which led to a proper diagnosis of $\mathrm{AM}^{6,11,12}$ Furthermore, Spuler et al. found that the routine use of Congo red-stained sections increased the frequency of a diagnosis of AM 10-fold. The present case illustrates the difficulty of differentiating AM from inflammatory myopathies: the first suspected diagnosis was inclusion body myositis. The presence of neonatal myosin in some fibers reflects the immaturity/regenerative activity of the muscle and is a sign of a pathological state in the muscle. COX-negative fibers are a common finding in IBM, but non-specific, as they are often seen in the muscle of aged people. AM was not suspected until amyloidosis was seen in the gastric mucosal biopsy. Congo red staining eventually showed vascular and extracellular amyloid accumulations in the original muscle biopsy sections, which led to a diagnosis of AM. The use of MRI for the evaluation of inflammatory myopathies and neuromuscular disorders has become increasingly common, especially in cases of polymyositis, ${ }^{8,9}$ and its usefulness for diagnosing AM has also been described. ${ }^{11}$ Hull et al. reported a case of a patient with the occurrence of abnormal subcutaneous fat in MRI and increased T2 signal intensity and minimal signal intensity alternation in the muscles, and established that these findings coupled with minimal muscular involvement constituted a unique guide to a proper diagnosis of primary AM. This is consistent with the previous MRI findings in 2 patients described by Metzler et al. In particular a hypointense reticular appearance for the subcutaneous fat in MRI distinguishes AM from many other neuromuscular conditions. ${ }^{12,13}$ Furthermore, in the case of the patient described by Hull et al., MRI also revealed decreased T1 signaling in the bone marrow, suggesting hematologic malignancies. In the present case the muscle MRI revealed evidence suggestive of atrophy and oedema in the muscles of the hips, thighs and calves. Nevertheless, as noticed after the patient's death, the MRI had been performed without using a muscle-specific protocol, so that the finding remained inconclusive. In summary, we have reported here on a well-described case of the rarely occurring disease AM. This case demonstrates that AM can masquerade as IBM, which is known to be a more common disorder. The inflammatory myopathies are increasingly being evaluated using MRI, which can be of practical significance in differentiating AM from other muscle diseases, targeting affected muscles for biopsy and assessing disease activity. ${ }^{12}$ In addition, MRI is of use when performed with an appropriate muscle-specific protocol. To clarify the etiological diagnosis of myopathy, however, Congo red staining and immunohistochemical testing should be performed.

\section{References}

1. Gertz MA, Kyle RA. Myopathy in primary systemic amyloidosis. J Neurol Neurosurg Psychiatry 1996;60:655-60.

2. Kyle RA, Gertz MA. Systemic amyloidosis. Crit Rev Oncol Hematol 1990;10:49-87.

3. Chapin JE, Kornfeld M, Harris A. Amyloid myopathy: characteristic features of a still underdiagnosed disease. Muscle Nerve 2005; 31:266-72.

4. Prayson RA. Amyloid myopathy: clinicopathologic study of 16 cases. Hum Pathol 1998; 29:463-8.

5. Rubin DI, Hermann RC. Electrophysiologic findings in amyloid myopathy. Muscle Nerve 1999; 22:355-9.

6. Karacostas D, Soumpourou M, Mavromatis I, et al. Isolated myopathy as the initial manifestation of primary systemic amyloidosis. J Neurol 2005; 252:853-4.

7. Spuler S, Emslie-Smith A, Engel AG. Amyloid myopathy. An underdiagnosed entity. Ann Neurol 1998; 43:719-28.

8. Adams EM, Chow CK, Premkumar A, Plotz PH. The idiopathic inflammatory myopathies: spectrum of MR imaging findings. Radiographics 1995; 15:563-74.

9. Mercuri E, Pichiecchio A, Allsop J, et al. Muscle MRI in inherited neuromuscular disorders: past, present, and future. J Magn Reson Imaging 2007; 25:433-40.

10. Lubarsch 0. Zur Kennthis ungewöhnlicher Amyloidablagerungen Virchows. Arch Pathol Anat Histol 1929;271:867-89.

11. Hull ME, Griffith L, Kuncl RW, Wigley FM. A deceptive case of amyloid myopathy. Clinical and magnetic resonance imaging features. Arthritis Rheum 2001; 8:1954-8.

12. Mandl LA, Folkerth RD, Pick MA, et al. Amyloid myopathy masquerading as polymyositis. J Rheumatol 2000; 27:94952.

13. Metzler JP, Fleckenstein JL, White CL, et al. MRI evaluation of amyloid myopathy. Skeletal Radiol 1992;21:463-5. 